\title{
Economics of Mental Health: Providing a Platform for Efficient Mental Health Policy
}

\author{
Christopher M. Doran ${ }^{1} \cdot$ Irina Kinchin ${ }^{2,3}$
}

Published online: 15 March 2020

○) Springer Nature Switzerland AG 2020

According to the World Health Organization (WHO), mental health is a state of wellbeing in which every individual realizes their potential, can cope with the normal stresses of life, can work productively and fruitfully, and is able to contribute to the community [1]. Unfortunately, problems associated with mental health are common in all regions of the world, affecting every community and age group across all-income countries [1].

Mental-health-related disorders account for an estimated $7 \%$ of all global disease as measured in disability-adjusted life-years, $19 \%$ of all years lived with disability [2], and $16 \%$ of the global burden of disease and injury in people aged 10-19 years [3]. Almost three quarters of the global burden is in low- and lower middle-income countries [1].

Mental ill-health also weighs heavily on societies and economies, with the annual cost of mental illness to developed countries estimated at $4 \%$ of gross domestic product and results in around 12 million days of reduced productivity each year [4]. There are significant social and economic benefits of improving individuals' mental health [5], yet mentalhealth-related disorders account for $<1 \%$ of most countries' healthcare budget [6].

An improvement in an individual's mental health can provide flow-on benefits in terms of increased social and economic participation, engagement and connectedness, and productivity in employment. This can in turn enhance the wellbeing of the wider community, including through

Christopher M. Doran

c.doran@cqu.edu.au

1 Cluster for Resilience and Wellbeing, Centre for Indigenous Health Equity Research, Central Queensland University, Brisbane, Australia

2 Centre for Health Economics Research and Evaluation and the Centre for Improving Palliative, Aged and Chronic Care Through Clinical Research and Translation, University of Technology Sydney, Ultimo, Australia

3 Global Brain Health Institute, Dublin, Ireland more rewarding relationships for family and friends; a lower burden on informal carers; a greater contribution to society through volunteering and working in community groups; increased output for the community from a more productive workforce; and an associated expansion in national income and living standards. These raise the capacity of the community to invest in interventions to improve mental health, thereby completing a positive reinforcing loop [5].

In acknowledgment of the importance of mental health, this themed issue of Applied Health Economics and Health Policy contains a series of 13 papers on the 'Economics of Mental Health'. From a quick glance at the articles, the reader will notice that the collection is diverse, representing high- to low- and middle-income countries, various population groups and canvassing a range of topics. The content is novel, relevant and timely with sound research and policy implications noted.

A contribution from Zweifel is based on the premise that patients with mental health problems are often inconsistent in the way in which they express their preferences, and that a decision rule can be devised to help identify agents who will act on patients' behalf to find out their true preferences. The decision rule includes five dimensions of preference: when treatment is to be initiated, what is the type of disorder, who is to provide treatment, how is it to proceed, and where it is to be performed. Using mathematical modelling to test the decision rule, Zweifel suggests that no single agent is found to dominate with respect to the five dimensions of preference distinguished, constituting a major challenge for economic theory and the evaluation of changes in policy [7].

Stoyanova and Pinilla examine the impact of economic volatility on mental health in Spain [8]. Using data from national health surveys, mental health is proxied by two measures, doctor-diagnosed mental disorder and psychological distress. The authors observe different patterns of the two mental health indicators over the 15 -year period of interest; psychological distress in men increased but slightly decreased among women; and diagnosed mental disorders 
declined during the peak years of the crisis. The authors suggest that policies aiming to tackle the challenges posed by the high prevalence of mental disorders have to be particularly attentive to changes in individuals' socioeconomic situation, including education, unemployment and social class [8].

Psychological distress was also examined by Chirwa et al., who used instrumental variable and propensity score matching methods to estimate the causal impact of health insurance on psychological distress in Ghana [9]. The study suggests that having health insurance is associated with reduced psychological distress and improved mental health, even though mental illness treatment or prevention were at best only partially covered by the National Health Insurance Scheme. The authors found the beneficial impact of health insurance on psychological distress is larger for wealthier than poorer insurance members, suggesting that there may be a need to facilitate access to health insurance for people on lower incomes [9].

Three of the papers in the special issue focus on the economic burden of mental health [10-12]. To inform ongoing mental healthcare reform in Czechia, Broulíková et al. used a bottom-up micro-costing approach to calculate the unit costs of inpatient and community mental health services [10]. An Australian study by Nghiem et al. estimates the costs of mental health in Australia using the standard-of-living (SoL) approach applied to longitudinal data [11]. The SoL approach estimates the cost of mental illness as the amount of additional income needed to make the standard of living of people with a mental illness like that of people without mental illness. The authors estimate that people with mental illness need to increase equivalized disposable income by $50-80 \%$ to achieve a similar level of financial satisfaction to those without a mental illness [11]. Ride et al. presents a costing methodology to estimate costs for people with serious mental illness in England [12]. The authors conducted a retrospective observational cohort study using linked administrative records from primary care, emergency departments, inpatient admissions, and community mental health services. The findings of this analysis are policy relevant in that they can help policymakers plan health budgets and inform policy levers to manage resource use across the care pathway [12].

Two papers focused on suicide prevention [13, 14]. Kinchin et al. used decision analytic modelling to examine the effectiveness of suicide prevention training (safeTALK) in an Australian school setting [13]. SafeTALK is a suicide prevention training programme developed by Living Works Education to help (a) recognize warning signs of suicide; (b) move beyond common tendencies to miss, dismiss, and avoid suicide; (c) notice and respond to situations in which thoughts of suicide might be present, apply basic TALK steps (Tell, Ask, Listen, and KeepSafe); and (d) connect the suicidal person with suicide first aid help and further community resources. The authors found the training was both effective and provided a positive return on investment [13]. A Canadian study by Lebenbaum et al. developed a Markov model to estimate the costs and effects of a suicide-prevention strategy in the province of Ontario. The strategy consisted of a package of interventions geared towards preventing suicide, including a public health awareness campaign, increased identification of individuals at risk, increased training of primary-care physicians, and increased treatment post-suicide attempt. The authors found the suicide-prevention campaign a cost-effective strategy to reduce the incidence of suicide [14].

Several articles focused on efforts to improve mental health care for vulnerable populations [15-18]. Cullinan et al. examined socioeconomic disparities in unmet need for student mental health services in higher education in Ireland [15]. The authors used three separate measures of mental health problems, namely stress, anxiety, and depression, to develop and model an indicator of unmet need [15]. The authors found large socioeconomic disparities in unmet need for mental health services and suggest that these disparities are driven by higher rates of mental ill-health among those from lower socioeconomic backgrounds [15].

A novel and timely contribution by Meads et al. examined the cost effectiveness of dementia care mapping (DCM) in care-home settings in the United Kingdom [16]. DCM is a care-home-level intervention that aims to support the embedding of person-centred care, improve the quality of care and, consequently, health outcomes for residents. Despite the potential scalability, the authors found that DCM was not cost effective compared with usual care in preventing or supporting agitation in care home residents with dementia. The study highlighted a number of challenges in conducting research in this population group, including dealing with low intervention compliance; high levels of missing data; uncertain validity of utility assessments in this population and high cost outliers [16].

Schmidt et al. conducted a systematic review of health economic evaluations of universal mental health Interventions for children and adolescents [17]. Only nine studies were included in the review, with considerable variation between studies regarding intervention aims, methodology and outcome measures, thereby limiting the generalizability of findings. The authors note that there is some evidence based on decision-analytic modelling that anti-bullying interventions represent value for money but further research is warranted [17].

Turner et al. undertook an economic evaluation of a school-based social and emotional learning Intervention in the United Kingdom [18]. The intervention, Promoting Alternative Thinking Strategies (PATHS), aim of to promote self-control, emotional understanding, positive selfesteem, relationships and interpersonal problem-solving 
skills among children in pre-school and primary education settings through the provision of a taught curriculum. The authors found that the PATHS curriculum had the potential to be cost effective at standard willingness-to-pay thresholds but more work was required before allocating scarce public resources [18].

One paper focused on issues associated with equity. Başar and Öztürk used a horizontal inequity (HI) index to investigate the degree of equity in mental healthcare services utilization in Turkey [19]. The HI index is derived from a national health survey for males and females. The authors report pro-poor inequity and gender variations and suggest that there is a greater need for gender-focused policies to improve horizontal equity in mental healthcare services utilization [19].

This themed issue on the 'Economics of Mental Health' has canvassed a range of current research on the topic. The consensus from each of the participating authors is that more needs to be done. Indeed, the WHO recommends that health spending should be proportionate to the need [1]. We applaud the contributors to the special issue and would encourage other researchers to take up the challenge to demonstrate the potential economic benefits of investing in mental health policies and programmes.

\section{Compliance with Ethical Standards}

Funding No funding was received to support the writing of this editorial.

Conflict of Interest Christopher Doran and Irina Kinchin have no conflict of interest directly related to this editorial.

\section{References}

1. World Health Organisation. Mental Health Action Plan 20132020. Geneva: WHO; 2013.

2. Rehm J, Shield K. Global burden of disease and the impact of mental and addictive disorders. Curr Psychiatry Rep. 2019. https ://doi.org/10.1007/s11920-019-0997-0.

3. World Health Organisation. Adolescent mental health Geneva: WHO; 2020. https://www.who.int/news-room/fact-sheets/detail/ adolescent-mental-health. Accessed 30 Jan 2020.

4. Organisation for Economic Co-operation and Development. Mental Health: OECD; 2019. https://www.oecd.org/health/health-syste ms/mental-health.htm. Accessed 30 Jan 2020.

5. Australian Government Productivity Commission. The Social and Economic Benefits of Improving Mental Health. Canberra: Productivity Commission; 2019.

6. World Health Organisation. mhGAP Mental Health Gap Action Programme Scaling up care for mental, neurological, and substance use disorders. Geneva: WHO; 2008.
7. Zweifel P. Mental health: a particular challenge confronting policy makers and economists. Appl Health Econ Health Policy. 2019. https://doi.org/10.1007/s40258-019-00479-2.

8. Stoyanova A, Pinilla J. The evolution of mental health in the context of transitory economic changes. Appl Health Econ Health Policy. 2019. https://doi.org/10.1007/s40258-019-00537-9.

9. Chirwa GC, Suhrcke M, Moreno-Serra R. The impact of Ghana's National Health Insurance on psychological distress. Appl Health Econ Health Policy. 2019. https://doi.org/10.1007/s40258-01900515-1.

10. Broulíková HM, Winkler P, Páv M, Kondrátová L. Costs of mental health services in Czechia: facilitating an evidence-based reform of psychiatric care. Appl Health Econ Health Policy. 2019. https ://doi.org/10.1007/s40258-019-00501-7.

11. Nghiem $S$, Khanam R, Vu XB, Tran BX. Implicitly estimating the cost of mental illness in Australia: a standard-of-living approach. Appl Health Econ Health Policy. 2019. https://doi.org/10.1007/ s40258-019-00526-y.

12. Ride J, Kasteridis P, Gutacker N, Aragon MJ, Jacobs R. healthcare costs for people with serious mental illness in England: an analysis of costs across primary care, hospital care, and specialist mental healthcare. Appl Health Econ Health Policy. 2019. https ://doi.org/10.1007/s40258-019-00530-2.

13. Kinchin I, Russell AM, Petrie D, Mifsud A, Manning L, Doran CM. Program Evaluation and Decision Analytic Modelling of Universal Suicide Prevention Training (safeTALK) in Secondary Schools. Appl Health Econ Health Policy. 2019. https://doi. org/10.1007/s40258-019-00505-3.

14. Lebenbaum M, Cheng J, de Oliveira C, Kurdyak P, Zaheer J, Hancock-Howard R, et al. Evaluating the cost effectiveness of a suicide prevention campaign implemented in Ontario, Canada. Appl Health Econ Health Policy. 2019. https://doi.org/10.1007/ s40258-019-00511-5.

15. Cullinan J, Walsh S, Flannery D. Socioeconomic disparities in unmet need for student mental health services in higher education. Appl Health Econ Health Policy. 2019. https://doi.org/10.1007/ s40258-019-00529-9.

16. Meads DM, Martin A, Griffiths A, Kelley R, Creese B, Robinson $\mathrm{L}$, et al. Cost-effectiveness of dementia care mapping in carehome settings: evaluation of a randomised controlled trial. Appl Health Econ Health Policy. 2019. https://doi.org/10.1007/s4025 8-019-00531-1.

17. Schmidt M, Werbrouck A, Verhaeghe N, Putman K, Simoens $\mathrm{S}$, Annemans L. Universal mental health interventions for children and adolescents: a systematic review of health economic evaluations. Appl Health Econ Health Policy. 2019. https://doi. org/10.1007/s40258-019-00524-0.

18. Turner AJ, Sutton M, Harrison M, Hennessey A, Humphrey N. Cost-effectiveness of a school-based social and emotional learning intervention: evidence from a cluster-randomised controlled trial of the promoting alternative thinking strategies curriculum. Appl Health Econ Health Policy. 2019. https://doi.org/10.1007/s4025 8-019-00498-z.

19. Başar D, Öztürk S. Assessing horizontal equity in the utilization of mental healthcare services in turkey: a gender perspective. Appl Health Econ Health Policy. 2019. https://doi.org/10.1007/s4025 8-019-00519-x. 